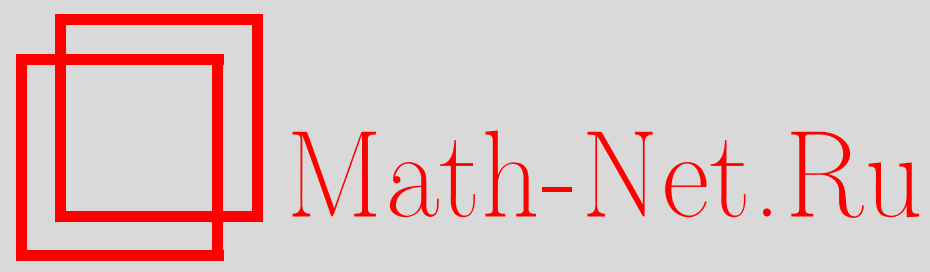

М. И. Тихомирова, Асимптотическая нормальность числа непоявившихся несплошных цепочек исходов независимых испытаний, Дискрет. матем., 2009, том 21, выпуск 2, 112 125

DOI: https://doi.org/10.4213/dm1051

Использование Общероссийского математического портала Math-Net.Ru подразумевает, что вы прочитали и согласны с пользовательским соглашением http://www .mathnet.ru/rus/agreement

Параметры загрузки:

IP : 52.90 .164 .192

26 апреля 2023 г., 13:28:41 


\title{
Асимптотическая нормальность числа непоявившихся несплошных цепочек исходов независимых испытаний
}

\author{
๑) 2009 г. М. И. Тихомирова
}

\begin{abstract}
Доказана асимптотическая нормальность числа непоявившихся несплошных цепочек исходов независимых испытаний. Асимптотическая нормальность числа непоявившихся цепочек одинаковых исходов, известная для равновероятных исходов, установлена в неравновероятном случае.
\end{abstract}

\section{1. Введение}

Обозначим через $\mu_{0}\left(\mathscr{B}_{N}\right)$ число непоявившихся $v$-цепочек из некоторого множества $\mathscr{B}_{N}$ в $n$ независимых испытаниях с $N$ исходами в каждом. Если $\mathscr{B}_{N}$ является множеством всех возможных значений $v$-цепочек, то его мощность $\left|\mathscr{B}_{N}\right|=N^{v}$. Асимптотическая нормальность $\mu_{0}\left(\mathscr{B}_{N}\right)$ для сплошных цепочек при $n, N \rightarrow \infty, 0<\alpha_{1} \leqslant n / N^{v} \leqslant \alpha_{2}<\infty$ была установлена в работах [1-3]. Доказательство основано на результатах работы [4] о разделимых статистиках, построенных по частотам сплошных цепочек исходов. По условиям теорем работы [4] требуется, чтобы дисперсия $\mathbf{D} \mu_{0}\left(\mathscr{B}_{N}\right)$ имела порядок $n$. При достаточно общих условиях $\mathbf{D} \mu_{0}\left(\mathscr{B}_{N}\right)$ имеет порядок $\left|\mathscr{B}_{N}\right|$. Таким образом, когда $\left|\mathscr{B}_{N}\right|$ имеет порядок, меньший $n$, асимптотическая нормальность $\mu_{0}\left(\mathscr{B}_{N}\right)$ из результатов работы [4] не следует.

В данной работе получены достаточные условия асимптотической нормальности $\mu_{0}\left(\mathscr{B}_{N}\right)$ в более общем случае несплошных цепочек исходов; результаты работы [5], относящиеся к сплошным цепочкам исходов в равновероятных испытаниях, перенесены на испытания с неравновероятными исходами.

\section{2. Постановка задачи}

Пусть

$$
x_{1}, x_{2}, \ldots, x_{t}, \ldots
$$

- независимые случайные величины с распределением вероятностей

$$
\mathbf{P}\left\{x_{t}=k\right\}=p_{k}, \quad k=1, \ldots, N, \quad t=1,2, \ldots
$$


По последовательности (1) образуется последовательность $v$-цепочек

$$
\bar{x}(t)=\left(x_{t+r_{1}-1}, x_{t+r_{2}-1}, \ldots, x_{t+r_{v}-1}\right), \quad t=1,2, \ldots, \quad 1=r_{1}<r_{2}<\ldots<r_{v}=s .
$$

При $v=s$ последовательность (2) есть последовательность сплошных $s$-цепочек.

Пусть $\mathscr{B}_{N}$ обозначает произвольное множество $v$-цепочек $I=\left(i_{1}, \ldots, i_{v}\right)$, где $i_{1}, \ldots, i_{v} \in\{1, \ldots, N\}$. Число $v$-цепочек из множества $\mathscr{B}_{N}$, не появившихся в $n$ первых членах последовательности (2), обозначим через $\mu_{0}\left(\mathscr{B}_{N}\right)$. Предельные распределения $\mu_{0}\left(\mathscr{B}_{N}\right)$ будем рассматривать в предположении, что при $N \rightarrow \infty$

$$
N p_{k} \leqslant A, \quad k=1, \ldots, N, \quad 0<\underline{\alpha} \leqslant \alpha_{n}=\frac{n}{N^{v}} \leqslant \bar{\alpha}<\infty,
$$

где $A>0, \underline{\alpha}, \bar{\alpha}-$ некоторые постоянные.

Случайную величину $\mu_{0}\left(\mathscr{B}_{N}\right)$, используя известные формулы для комбинаций событий (см. [6]), можно представить в виде суммы зависимых случайных величин. Методы исследования сумм зависимых случайных величин, предложенные С. Н. Бернштейном (см. [7]), успешно использовались, когда зависимость слагаемых удобно было связать с разностью номеров слагаемых. Более сложные виды зависимостей оказалось удобнее описывать при помощи специально подобранных графов (см. [8]). Условия теоремы Янсона об асимптотической нормальности [8] легко проверяются. В. Г. Михайлов (см. [9]), допустив усложнение условий теоремы Янсона, получил более общее утверждение без существенного изменения доказательства.

В данной работе используется представление $\mu_{0}\left(\mathscr{B}_{N}\right)$ в виде суммы зависимых величин. В разделе 2 показано, что в этой сумме можно пренебречь частью слагаемых, а оставшуюся часть рассматривать как аппроксимирующую сумму, предельное распределение которой совпадает с предельным распределением $\mu_{0}\left(\mathscr{B}_{N}\right)$. В разделе 3 получены достаточные условия асимптотической нормальности $\mu_{0}\left(\mathscr{B}_{N}\right)$ (см. теорему 2 ). Применение этой теоремы иллюстрируется доказательством асимптотической нормальности $\mu_{0}\left(\mathscr{B}_{N}\right)$ в двух важных частных случаях:

(1) $v=s, \quad \mathscr{B}_{N}=\left\{I: i_{1}=\ldots=i_{N}=i, i=1, \ldots, N\right\}$,

(2) $v=3, \quad r_{2}=r, \quad r_{3}=s, \quad \mathscr{B}_{N}=\left\{I=\left(i_{1}, i_{2}, i_{3}\right): i_{k}=1, \ldots, N, k=1,2,3\right\}$.

Для доказательства асимптотической нормальности аппроксимирующих сумм используем утверждение, непосредственно вытекающее из результатов работ [8, 9]. Приведем это утверждение в удобном для дальнейшего использования виде.

Введем сначала несколько обозначений. Вектор номеров членов последовательности (1), соответствующих координатам вектора $\bar{x}(t)$, обозначим через $\tau(t)$,

$$
\tau(t)=\left(t+r_{1}-1, t+r_{2}-1, \ldots, t+r_{v}-1\right) .
$$

Иногда удобно вектор $\tau(t)$ рассматривать как множество его координат. Будем говорить, что векторы $\bar{x}\left(t_{1}\right)$ и $\bar{x}\left(t_{2}\right)$ или $\tau\left(t_{1}\right)$ и $\tau\left(t_{2}\right)$ пересекаются или сцеплены, если $\tau\left(t_{1}\right) \cap \tau\left(t_{2}\right) \neq \varnothing$.

Рассмотрим множество пар $\gamma=\left(u ;\left(t_{1}, \ldots, t_{u}\right)\right)$. Определим множества $\mathscr{D}_{N}(l)$, $\Gamma_{N}\left(L_{N}\right)$, полагая

$$
\mathscr{D}_{N}(l) \subseteq\left\{\gamma: u=l, 1 \leqslant t_{1}<\ldots<t_{l} \leqslant n\right\}, \quad \Gamma_{N}\left(L_{N}\right)=\bigcup_{l=1}^{L_{N}} \mathscr{D}_{N}(l) .
$$


Приведем условия асимптотической нормальности сумм

$$
S_{N}=\sum_{\gamma \in \Gamma_{N}\left(L_{N}\right)} \eta_{\gamma}
$$

где $\eta_{\gamma}=g_{l}\left(\bar{x}\left(t_{1}\right), \ldots, \bar{x}\left(t_{l}\right)\right),\left|g_{l}\left(\bar{x}\left(t_{1}\right), \ldots, \bar{x}\left(t_{l}\right)\right)\right| \leqslant 1$ при любых значениях $\bar{x}\left(t_{k}\right)$, $k=1, \ldots, l$; векторы $\bar{x}(t)$ определены равенствами (1) и (2). Положим

$$
V \subset \Gamma_{N}\left(L_{N}\right), \quad G(\gamma)=\bigcup_{k=1}^{u} \tau\left(t_{k}\right), \quad G_{V}=\bigcup_{\gamma \in V} G(\gamma) .
$$

Определим множество $F_{V}$, полагая

$$
F_{V}=\left\{\gamma: \text { найдется } \gamma^{\prime} \in V \text { такое, что } G(\gamma) \cap G\left(\gamma^{\prime}\right) \neq \varnothing\right\} \text {. }
$$

Отметим, что $x_{t} \mathrm{c} t \notin F_{V}$ не зависят от $\left\{x_{t}: t \in V\right\}$.

Будем предполагать, что при любом натуральном $m$ найдется такая величина $Q_{m}$, не зависящая от $V$, что при любом $V$ с $|V| \leqslant m$

$$
H_{V}=\sum_{\gamma \in F_{V}} \mathbf{E}\left(\left|\eta_{\gamma}\right| \mid x_{t}, t \in G_{V}\right) \leqslant Q_{m}
$$

при любых значениях $x_{t}, t \in G_{V}$.

Теорема 1. Если при $N \rightarrow \infty$ выполняются условия (3), (6) и при некотором $\beta$, $0<\beta \leqslant 2 / 3$, выполняются условия

$$
\left|\Gamma_{N}\left(L_{N}\right)\right| \rightarrow \infty, \quad \frac{M_{N}^{\beta} Q_{m}^{2-\beta}}{\mathbf{D} S_{N}} \rightarrow 0,
$$

2de

$$
M_{N}=\sum_{\gamma \in \Gamma_{n}\left(L_{N}\right)} \mathbf{E}\left|\eta_{\gamma}\right|
$$

то распределение величинь $\left(S_{N}-\mathbf{E} S_{N}\right) / \sqrt{\mathbf{D} S_{N}}$ сходится к стандартному нормальному распределению.

Эта теорема непосредственно следует из результатов работ $[8,9]$.

\section{3. Аппроксимирующие суммы}

Число $\mu_{0}\left(\mathscr{R}_{N}\right)$ непоявившихся $v$-цепочек в $n$ первых членах последовательности (2) можно представить в виде

$$
\mu_{0}\left(\mathscr{B}_{N}\right)=\sum_{I \in \mathscr{B}_{N}} \chi(\bar{x}(t) \neq I, 1 \leqslant t \leqslant n), \quad I=\left(i_{1}, \ldots, i_{v}\right),
$$

где $\chi(A)-$ индикатор события $A$.

Для любых событий $A_{1}, \ldots, A_{n}$ и любого распределения вероятностей (см. [6])

$$
\mathbf{P}\left(\bar{A}_{1} \ldots \bar{A}_{n}\right)=\sum_{l=0}^{n}(-1)^{l} \sum_{\bar{t}(l) \in T(l)} \mathbf{P}\left(A_{t_{1}} \ldots A_{t_{l}}\right),
$$


где $\bar{t}(l)=\left(t_{1}, \ldots, t_{l}\right), T(l)=\left\{\bar{t}(l): 1 \leqslant t_{1}<\ldots<t_{l} \leqslant n\right\}$. Для величины $\Delta_{\varphi}^{*}$, равной

$$
\Delta_{\varphi}^{*}=\mathbf{P}\left(\bar{A}_{1} \ldots \bar{A}_{n}\right)-\sum_{l=0}^{\varphi-1}(-1)^{l} \sum_{\bar{t}(l) \in T(l)} \mathbf{P}\left(A_{t_{1}} \ldots A_{t_{l}}\right),
$$

при любом $\varphi$ выполняется неравенство

$$
\left|\Delta_{\varphi}^{*}\right| \leqslant \sum_{\bar{t}(\varphi) \in T(\varphi)} \mathbf{P}\left(A_{t_{1}} \ldots A_{t_{\varphi}}\right) .
$$

Положим $A_{t}=\{\bar{x}(t)=I\}$. Если заданы $n+s-1$ первых членов последовательности (1), $w=\left(x_{1}, \ldots, x_{n+s-1}\right)$, то условные вероятности событий, входящих в (9) и (10), можно представить в виде

$$
\begin{aligned}
\mathbf{P}\left(\bar{A}_{1} \ldots \bar{A}_{n} \mid w\right) & =\chi(\bar{x}(t) \neq I, t=1, \ldots, n), \\
\mathbf{P}\left(A_{t_{1}} \ldots A_{t_{l}} \mid w\right) & =\chi\left(\bar{x}\left(t_{1}\right)=\ldots=\bar{x}\left(t_{l}\right)=I\right) .
\end{aligned}
$$

Отсюда и из равенств (8), (9) и (10) получаем, что

$$
\mu_{0}\left(\mathscr{B}_{N}\right)=\sum_{l=0}^{\varphi-1} \sum_{\bar{t}(l) \in T(l)} \xi_{l}(\bar{t}(l))+\Delta_{\varphi},
$$

где

$$
\begin{gathered}
\xi_{l}(\bar{t}(l))=(-1)^{l} \sum_{I \in \mathscr{B}_{N}} \chi\left(\bar{x}\left(t_{1}\right)=\ldots=\bar{x}\left(t_{l}\right)=I\right), \\
\Delta_{\varphi}=\sum_{l=\varphi}^{n} \sum_{\bar{t}(l) \in T(l)} \xi_{l}\left(t_{1}, \ldots, t_{l}\right), \quad\left|\Delta_{\varphi}\right| \leqslant \sum_{\bar{t}(\varphi) \in T(\varphi)}\left|\xi_{\varphi}(\bar{t}(\varphi))\right| .
\end{gathered}
$$

В сумме (11) выделим слагаемые, которыми можно пренебречь. Представим множество $T(l)$ в виде объединения непересекающихся множеств

$$
T(l)=T_{1}(l) \cup T_{2}(l) \cup T_{3}(l),
$$

где

$$
T_{1}(l)=\left\{\left(t_{1}, \ldots, t_{l}\right): \tau\left(t_{i}\right) \cap \tau\left(t_{j}\right)=\varnothing \text { при любых } i \neq j\right\} ;
$$

множество $T_{2}(l)$ включает такие $\left(t_{1}, \ldots, t_{l}\right)$, для которых среди множеств $\tau\left(t_{1}\right), \ldots, \tau\left(t_{l}\right)$ найдется ровно одна пара пересекающихся множеств, а множество $T_{3}(l)$ включает остальные $\left(t_{1}, \ldots, t_{l}\right)$. Аналогичное представление $T(l)$ использовалось в [2] для сплошных цепочек.

Представим теперь $\mu_{0}\left(\mathscr{B}_{N}\right)$ в виде

$$
\mu_{0}\left(\mathscr{B}_{N}\right)=\mu_{1}(N)+\mu_{2}(N)+\mu_{3}(N)+\Delta_{\varphi},
$$

где $\Delta_{\varphi}$ определено равенством (13),

$$
\begin{aligned}
\mu_{u}(N) & =\sum_{l=0}^{\varphi-1} \sum_{\bar{t}(l) \in T_{u}(l)} \xi_{l}\left(t_{1}, \ldots, t_{l}\right), & & u=1,2,3, \\
\varphi & =[v(1+\delta) \ln N / \ln \ln N], & & \delta>0 .
\end{aligned}
$$


Из неравенства (10) и равенств (11)-(13) следует, что с вероятностью, равной единице,

$$
\left|\Delta_{\varphi}\right| \leqslant \sum_{\bar{t}(\varphi) \in T(\varphi)}\left|\xi_{\varphi}\left(t_{1}, \ldots, t_{\varphi}\right)\right|=\sum_{u=1}^{3} \bar{\Delta}_{u}, \quad \bar{\Delta}_{u}=\Delta_{u}(\varphi),
$$

где

$$
\Delta_{u}(l)=\sum_{\bar{t}(l) \in T_{u}(l)}\left|\xi_{l}\left(t_{1}, \ldots, t_{l}\right)\right|
$$

Следующие леммы 1 и 2, содержащие оценки $\mathbf{E} \Delta_{l}(u), u=1,2,3$, доказываются аналогично леммам 1-3 работы [2].

Лемма 1. При любом множестве $\mathscr{B}_{N}$ в условиях (3) выполняются неравенства

$$
\mathbf{E} \Delta_{1}(l) \leqslant \frac{\left(\alpha_{N} A^{v}\right)^{l}}{l !}\left|\mathscr{B}_{N}\right|, \quad \mathbf{E} \Delta_{2}(l) \leqslant C l(l-1) \frac{\left(\alpha_{N} A^{v}\right)^{l}}{l !}, \quad \mathbf{E} \Delta_{3}(l) \leqslant C \frac{c_{0}^{l}}{N},
$$

где $C>0, c_{0}>0$ - некоторые постоянные, величина А входит в условия (3).

Доказательство. Доказательство первого неравенства совпадает с соответствующей частью доказательства леммы 1 работы [2]. Доказательство третье неравенства с незначительными изменениями повторяет доказательства лемм 1 и 3 работы [2]. Эти изменения относятся к поправкам оценок числа слагаемых, содержащих сцепленные несплошные цепочки и влияют только на величину постоянных $c_{0}$ и $C$. Остановимся подробнее на втором неравенстве.

Отметим, что при сплошных цепочках две сцепленные несовпадающие цепочки имеют следующий вид:

$$
\begin{aligned}
\bar{x}(t) & =\left(x_{t}, \ldots, x_{t+s-1}\right), \\
\bar{x}(t+s-u) & =\left(x_{t+s-u}, \ldots, x_{t+2 s-u-1}\right), \quad u=1,2, \ldots, s-1 .
\end{aligned}
$$

У этих цепочек ровно $u$ общих членов. У несплошных цепочек

$$
\begin{aligned}
\bar{x}(t) & =\left(x_{t+r_{1}-1}, \ldots, x_{t+r_{v}-1}\right), \\
\bar{x}\left(t+r_{v}-a\right) & =\left(x_{t+r_{v}-a+r_{1}-1}, \ldots, x_{t+2 r_{v}-a-1}\right)
\end{aligned}
$$

число $u$ общих членов существенно зависит от чисел $\left(r_{1}, \ldots, r_{v}\right)$. Цепочки не пересекаются при $a \geqslant r_{v}=s$, но могут не пересекаться и при $a<r_{v}$.

Так же, как и при доказательстве леммы 2 работы [2], оценим сначала вероятность $\mathbf{P}\left(\bar{x}\left(t_{1}\right)=\ldots=\bar{x}\left(t_{l}\right)=I\right)$, когда сцеплены цепочки $\bar{x}\left(t_{1}\right), \bar{x}\left(t_{2}\right)$ и число общих членов последовательности (1) в этих цепочках равно $u$. Из условий $(3)$ при $\left(t_{1}, \ldots, t_{l}\right) \in T_{2}(l)$ следует, что

$$
\begin{aligned}
\mathbf{P}\left\{\bar{x}\left(t_{1}\right)=\ldots=\bar{x}\left(t_{l}\right)=I\right\} & =\mathbf{P}\left\{\bar{x}\left(t_{1}\right)=\bar{x}\left(t_{2}\right)=I\right\} \\
& =\mathbf{P}\left\{\bar{x}\left(t_{3}\right)=\ldots=\bar{x}\left(t_{l}\right)=I\right\} \leqslant\left(\frac{A}{N}\right)^{2 v-u}\left(\frac{A^{v}}{N^{v}}\right)^{l-2} .
\end{aligned}
$$

В этом случае число наборов $I$, при которых эти вероятности отличны от 0 (пересечение цепочек не приводит к противоречию), при любом $\mathscr{B}_{N}$ не превосходит $N^{v-u}$; число различных значений $u$ не превосходит $s-1$ и каждое может встретиться в данной паре 
цепочек не более $v$ раз. Число слагаемых в сумме, определяющей $\Delta_{l}(2)$ с фиксированной разностью $t_{2}-t_{1}$, не превосходит $\left(\begin{array}{c}n \\ l-1\end{array}\right) \leqslant n^{l-1} /(l-1)$ !; число способов выбора сцепленной пары не превосходит $(l-1) s$. Из этих замечаний и условий (3) следует, что

$$
\begin{aligned}
\mathbf{E} \Delta_{2}(l) & \leqslant s^{2} v(v-1) \frac{n^{l-1}}{(l-1) !}\left(\frac{A}{N}\right)^{2 v-u}\left(\frac{A^{v}}{N^{v}}\right)^{l-2} N^{v-u} \\
& =s^{2} v(l-1)\left(\frac{A^{v} n}{N^{v}}\right)^{l-1} \frac{1}{(l-1) !} .
\end{aligned}
$$

Отсюда следует второе неравенство леммы 1.

Лемма 2. Если выполнены условия (3) и ө определено равенством (17), то при любом $\mathscr{B}_{N}$

$$
\begin{aligned}
& \mathbf{E} \Delta_{1}(\varphi) \leqslant C_{1} N^{-v \Delta\left(1+\gamma_{1}(N)\right)}, \\
& \mathbf{E} \Delta_{2}(\varphi) \leqslant C_{2} N^{-v(1+\Delta)\left(1+\gamma_{2}(N)\right)}, \\
& \mathbf{E} \Delta_{3}(\varphi) \leqslant C_{3} N^{-\left(1+\gamma_{3}(N)\right)},
\end{aligned}
$$

где $\gamma_{k}(N)=O\left(h_{N}\right), k=1,2,3, h_{N}=\ln \ln \ln N / \ln \ln N, C_{1}, C_{2}, C_{3}$ - некоторые положительные постоянные.

Доказательство. Неравенства леммы 2 следуют из неравенств леммы 1, неравенства $\left|\mathscr{B} c_{N}\right| \leqslant N^{v}$, равенства (21) и равенств

$$
\begin{aligned}
\ln \varphi & =\ln \ln N+O(\ln \ln \ln N), \\
\varphi \ln \varphi & =v(1+\delta) \ln N\left(1+O\left(h_{N}\right)\right) .
\end{aligned}
$$

Оценим теперь величины

$$
\underline{\Delta}_{u}=\sum_{l=0}^{\varphi-1} \Delta_{u}(l), \quad u=2,3,
$$

где $\Delta_{u}(l)$ определены равенством (19).

Лемма 3. Если выполнены условия (3) и ө определено равенством (17), то при любых $\mathscr{B}_{N}$ u $N$

$$
\mathbf{E} \underline{\Delta}_{2} \leqslant C, \quad \quad \mathbf{E}_{3} \leqslant C N^{-(1+O(1 / \ln \ln N))},
$$

где $C$ - некоторая положительная постоянная.

Утверждение этой леммы следует из леммы 1 и неравенств

$$
\begin{aligned}
& \mathbf{E} \underline{\Delta}_{2}=\sum_{l=0}^{\varphi-1} \mathbf{E} \Delta_{2}(l) \leqslant C \sum_{l=0}^{\varphi-1} l(l-1) \frac{\left(\alpha_{N} A^{v}\right)^{l}}{l !} \leqslant C, \\
& \mathbf{E} \underline{\Delta}_{3}=\sum_{l=0}^{\varphi-1} \mathbf{E} \Delta_{3}(l) \leqslant \frac{C}{N} \sum_{l=0}^{\varphi-1} c_{0}^{l} \leqslant \frac{C}{N} c_{0}^{\varphi} \leqslant C\left(\frac{1}{N}\right)^{1+O(\ln \ln N / \ln N)} .
\end{aligned}
$$

Следствие 1. При $N \rightarrow \infty$ в схеме серий (3) при любой функичи $f(N) \rightarrow \infty$ случайные величины

$$
\frac{\Delta_{2}}{f(N)}, \quad \frac{\underline{\Delta}_{3}}{f(N)}, \quad \frac{\Delta_{\varphi}}{f(N)}, \quad \frac{\mu_{0}\left(\mathscr{B}_{N}\right)-\mu_{1}(N)}{f(N)}
$$

сходятся по вероятности к 0. 
Это утверждение следует из леммы 3, леммы 2, неравенства (18) и неравенств

$$
\begin{array}{cl}
\mathbf{P}\left\{\frac{\underline{\Delta}_{u}}{f(N)}>\varepsilon\right\} \leqslant \frac{\mathbf{E} \Delta_{u}}{\varepsilon f(N)}, & u=2,3 ; \\
\mathbf{P}\left\{\frac{\Delta_{u}(\varphi)}{f(N)}>\varepsilon\right\} \leqslant \frac{\mathbf{E} \Delta_{u}(\varphi)}{\varepsilon f(N)}, & u=1,2,3 .
\end{array}
$$

Используя следствие 1 , неравенства $\left|\mu_{u}(N)\right| \leqslant \underline{\Delta}(u), u=2,3$, и неравенство (15), получаем следующее утверждение.

Следствие 2. Если в схеме серий (3) $\mathbf{D} \mu_{1}(N) \rightarrow \infty$ при $N \rightarrow \infty$, то

$$
\frac{\mu_{0}\left(\mathscr{B}_{N}\right)-\mu_{1}\left(\mathscr{B}_{N}\right)}{\sqrt{\mathbf{D} \mu_{1}(N)}} \rightarrow 0
$$

по вероятности.

Сумма $\mu_{1}(N)$ оказалась аппроксимирующей для $\mu_{0}\left(\mathscr{B}_{N}\right)$. Положим

$$
\mu_{0}^{*}\left(\mathscr{B}_{N}\right)=\mu_{1}(N) \text {. }
$$

Таким образом, для доказательства асимптотической нормальности $\mu_{0}\left(\mathscr{B}_{N}\right)$ нужно доказать асимптотическую нормальность $\mu_{1}(N)$ и то, что $\mathbf{D} \mu_{1}(N) \rightarrow \infty$.

\section{4. Достаточные условия асимптотической нормальности}

Будем предполагать, что в схеме серий (3) при $N \rightarrow \infty$

$$
0<b_{1} \leqslant \frac{\mathbf{D} \mu_{0}^{*}\left(\mathscr{B}_{N}\right)}{\left|\mathscr{B}_{N}\right|} \leqslant b_{2}<\infty, \quad\left|\mathscr{B}_{N}\right| \geqslant \varepsilon N, \quad 0<\varepsilon<1 .
$$

Теорема 2. В схеме серий (3) распределение случайной величины

$$
\frac{\mu_{1}\left(\mathscr{B}_{N}\right)-\mathbf{E} \mu_{1}(N)}{\sqrt{\mathbf{D} \mu_{1}(N)}}
$$

при $N \rightarrow \infty$ сходится к стандартному нормальному распределению, если выполнень условия (23).

Доказательство. В силу следствия 2, достаточно доказать асимптотическую нормальность величины $\left(\mu_{0}^{*}\left(\mathscr{\Re}_{N}\right)-\mathbf{E} \mu_{0}^{*}\left(\mathscr{B}_{N}\right)\right) / \sqrt{\mathbf{D} \mu_{0}^{*}\left(\mathscr{\Re}_{N}\right)}$.

Проверим выполнение условий теоремы 1 для сумм $S_{N}=\mu_{1}(N)$ с $L_{N}=\varphi-1$, $\gamma=\left(1,\left(t_{1}, \ldots, t_{l}\right)\right), \eta_{\gamma}=\xi_{\gamma}\left(t_{1}, \ldots, t_{l}\right)$ :

$$
S_{N}=\sum_{l=0}^{\varphi-1} \sum_{\bar{t}(l) \in T_{1}(l)} \xi_{l}\left(t_{1}, \ldots, t_{l}\right) .
$$

Из несовместности событий $\left(\bar{x}\left(t_{1}\right)=\ldots=\bar{x}\left(t_{l}\right)=I\right), I \in \mathscr{B}_{N}$, следует, что

$$
\left|\xi_{l}\left(t_{1}, \ldots, t_{l}\right)\right|=\sum_{I \in \mathscr{B}_{N}} \chi\left(\bar{x}\left(t_{1}\right)=\ldots=\bar{x}\left(t_{l}\right)=I\right) \leqslant 1 .
$$


Оценим величину

$$
M_{N}=\sum_{l=0}^{\varphi-1} \sum_{\bar{t}(l) \in T_{1}(l)} \sum_{I \in \mathscr{B}_{N}} \mathbf{E} \chi\left(\bar{x}\left(t_{1}\right)=\ldots=\bar{x}\left(t_{l}\right)=I\right) .
$$

Из независимости векторов $\bar{x}\left(t_{1}\right), \ldots, \bar{x}\left(t_{l}\right)$ следует, что

$$
\sum_{I \in \mathscr{B}_{N}} \mathbf{E} \chi\left(\bar{x}\left(t_{1}\right)=\ldots=\bar{x}\left(t_{l}\right)=I\right)=\sum_{I \in \mathscr{P}_{N}} \prod_{k=1}^{l} \mathbf{P}\left(\bar{x}\left(t_{k}\right)=I\right) \leqslant\left(\frac{A^{v}}{N^{v}}\right)^{l}\left|\mathscr{B}_{N}\right| .
$$

Мощность $\left|T_{1}(l)\right|$ множества $T_{1}(l)$ не превосходит $\left(\begin{array}{c}n \\ l\end{array}\right) \leqslant n^{l} / l$ !. Отсюда и из оценки (25) получаем, что

$$
M_{N} \leqslant\left|\mathscr{B}_{N}\right| \sum_{l=0}^{\varphi-1} \frac{n^{l}}{l !}\left(\frac{A^{v}}{N^{v}}\right)^{l} \leqslant\left|\mathscr{B}_{N}\right| \sum_{l=0}^{\infty} \frac{\left(\bar{\alpha} A^{v}\right)^{l}}{l !} \leqslant C\left|\mathscr{B}_{N}\right|,
$$

где $C$ - некоторая положительная постоянная.

Проверим условие (6). Выберем множество $V$ следующим образом:

$$
V=\left\{\left(l_{k},\left(t_{1}^{(k)}, \ldots, t_{l_{k}}^{(k)}\right)\right), k=1, \ldots, m_{0}\right\},
$$

где $l_{1}=\ldots=l_{m_{0}}=\varphi-1, m_{0}=m$. Полученные ниже оценки левой части (6) сохранятся, если все или часть $l_{k}$ будут меньше $\varphi-1$ и $m_{0}<m$. Множество $F_{V}$ определяется равенством (5). Оценим сначала слагаемые в левой части (6),

$$
E_{l}(k)=\mathbf{E}\left(\left|\xi_{l}\left(t_{1}, \ldots, t_{l}\right)\right|: x_{t} \in G_{V}\right)=\sum_{I \in \mathscr{B}_{N}} \mathbf{E} \chi\left(\bar{x}\left(t_{1}\right)=\ldots=\bar{x}\left(t_{l}\right)=I \mid x_{t} \in G_{V}\right),
$$

у которых среди $l$ цепочек с началами $t_{1}, \ldots, t_{l}$ ровно $k$ цепочек, например, цепочки $t_{1}, \ldots, t_{k}$, содержат $x_{t}$ с $t \in G_{V}$. Зацепившиеся с $G_{V}$ цепочки независимы между собой и не зависят от $x_{t}, t \in G_{V}$. Таким образом,

$$
E_{l}(k) \leqslant\left(\frac{A}{N^{v}}\right)^{l-k} \sum_{I \in \mathscr{B}_{N}} \mathbf{P}\left\{\bar{x}\left(t_{1}\right)=\ldots=\bar{x}\left(t_{k}\right)=I \mid x_{t} \in G_{V}\right\} \leqslant\left(\frac{A}{N^{v}}\right)^{l-k} .
$$

Число способов выбора цепочек $\bar{x}(t)$, у которых одной из координат является $x_{t_{0}}$, не превосходит $2 s-1$. Множество $G_{V}$ содержит не более $m(\varphi-1) v$ точек. Таким образом, число способов выбора начал $t_{1}, \ldots, t_{k}$ цепочек, каждая из которых среди координат имеет координату $x_{t}$ с $t \in G_{V}$, не превосходит $\left(\begin{array}{l}L \\ k\end{array}\right), L=(2 s-1)(\varphi-1) v m$; число способов выбора начал $t_{k+1}, \ldots, t_{l}$ цепочек, у которых нет координаты $x_{t} \mathrm{c} t \in G_{V}$, не превосходит $n^{l-k} /(l-k)$ !; число способов выбора $k$ сцепленных цепочек из $l$ цепочек равно $\left(\begin{array}{l}l \\ k\end{array}\right)$. Из сделанных замечаний и оценки (27) следует оценка левой части (6):

$$
H_{V} \leqslant \sum_{l=1}^{\varphi-1} \sum_{k=1}^{l}\left(\begin{array}{l}
l \\
k
\end{array}\right)\left(\begin{array}{l}
L \\
k
\end{array}\right) \frac{n^{l-k}}{(l-k) !}\left(\frac{\bar{\alpha} A^{v}}{N^{v}}\right)^{l-k}=\sum_{l=1}^{\varphi-1} \sum_{k=1}^{l}\left(\begin{array}{l}
l \\
k
\end{array}\right)\left(\begin{array}{l}
L \\
k
\end{array}\right) \frac{\left(\bar{\alpha} A^{v}\right)^{l-k}}{(l-k) !}
$$

Отсюда, меняя порядок суммирования по $l$ и $k$, получим оценку

$$
H_{V} \leqslant \sum_{k=1}^{\varphi-1}\left(\begin{array}{l}
L \\
k
\end{array}\right) R_{k},
$$


где

$$
R_{k}=\sum_{l=k}^{\varphi-1}\left(\begin{array}{l}
l \\
k
\end{array}\right) \frac{\left(\bar{\alpha} A^{v}\right)^{l-k}}{(l-k) !}=\sum_{l=0}^{\varphi-k-1}\left(\begin{array}{c}
l+k \\
k
\end{array}\right) \frac{\left(\bar{\alpha}_{N} A^{v}\right)^{l}}{l !} .
$$

Используя неравенства

$$
\begin{gathered}
\left(\begin{array}{c}
l+k \\
k
\end{array}\right)<\left(\begin{array}{l}
\varphi \\
k
\end{array}\right)<\varphi^{k} / k !, \quad l \leqslant \varphi-k-1, \\
R_{k}<C_{2} \frac{\varphi^{k}}{k !} \sum_{l=0}^{\varphi-k-1} \frac{\left(\bar{\alpha} A^{v}\right)^{l}}{l !} \leqslant C_{3} \varphi^{k} / k !,
\end{gathered}
$$

где $C_{2}, C_{3}$ - некоторые положительные постоянные, получим, что

$$
H_{V} \leqslant C_{3} \sum_{k=1}^{\varphi-1}\left(\begin{array}{l}
L \\
k
\end{array}\right) \frac{\varphi^{k}}{k !} \leqslant C_{3} \sum_{k=0}^{\varphi-1} \frac{\left(\varphi^{2} C_{1}^{2}\right)^{k}}{(k !)^{2}} \leqslant C_{3} \frac{\left(\varphi^{2} C_{1}^{2}\right)^{\varphi}}{(\varphi !)^{2}}
$$

Отсюда следует, что

$$
H_{V} \leqslant c_{m}^{\varphi}=Q_{m}
$$

где $c_{m}$ - некоторая положительная постоянная при заданном $m$. Проверим условия (7). Используя формулы (23), (26), (28) и (17), при $N \rightarrow \infty$ получим, что

$$
\frac{M_{N}^{\beta} Q_{m}^{2-\beta}}{\left|\mathscr{B}_{N}\right|} \leqslant C \frac{\left|\mathscr{B}_{N}\right|^{\beta} c_{m}^{\varphi(2-\beta)}}{\left|\mathscr{B}_{N}\right|} \leqslant \frac{N^{(2-\beta) /(\ln \ln N) \ln c_{0}}}{\left|\mathscr{B}_{N}\right|^{1-\beta}} \rightarrow 0
$$

где $C$ - некоторая положительная постоянная. Теорема доказана.

\section{5. Асимптотические формулы для моментов}

Приведем асимптотические формулы для среднего и дисперсии $\mu_{0}\left(\mathscr{B} c_{N}\right)$ в случаях 1 и 2 , указанных в разделе 1 . Множества $\mathscr{B}_{N}$ в этих двух частных случаях обозначим через $D_{1}$ и $D_{2}$ соответственно:

$$
\begin{aligned}
& D_{1}=\left\{I=\left(i_{1}, \ldots, i_{s}\right): i_{1}=\ldots=i_{s}=i, i=1, \ldots, N\right\}, \\
& D_{2}=\left\{I=\left(i_{1}, i_{2}, i_{3}\right): i_{k}=1, \ldots, N, k=1,2,3\right\} .
\end{aligned}
$$

Случайные величины $\mu_{0}\left(\mathscr{B}_{N}\right)$ и $\mu_{0}^{*}\left(\mathscr{B}_{N}\right)$ в этих двух случаях обозначим через $\mu_{0}\left(D_{1}\right)$, $\mu_{0}\left(D_{2}\right)$ и $\mu_{0}^{*}\left(D_{1}\right), \mu_{0}^{*}\left(D_{2}\right)$. Для сплошных цепочек асимптотические формулы получены в [2]. В рассматриваемом случае при $N \rightarrow \infty$, когда выполнены условия (3), эти формулы имеют следующий вид:

$$
\begin{aligned}
& \mathbf{E} \mu_{0}\left(D_{1}\right)=N A_{1}(1)+\alpha_{N}\left(\frac{1}{N} \sum_{i=1}^{N}\left(N p_{i}\right)^{s+1} e^{-\alpha_{N}^{*}\left(N p_{i}\right)^{s}}\right)+O\left(\frac{1}{N^{1-\delta}}\right), \\
& \mathbf{D} \mu_{0}\left(D_{1}\right)=N \sigma_{N}^{2}\left(D_{1}\right)+O(1), \quad 0<\delta<1,
\end{aligned}
$$


где

$$
\sigma_{N}^{2}\left(D_{1}\right)=A_{1}(1)-A_{2}(1), \quad A_{u}(1)=\frac{1}{N} \sum_{i=1}^{N} e^{-u \alpha_{N}^{*}\left(N p_{i}\right)^{s}}, \quad u=1,2 .
$$

В равновероятном случае, когда $p_{k}=1 / N, k=1, \ldots, N$,

$$
\begin{aligned}
& \mathbf{E} \mu_{0}\left(D_{1}\right)=N e^{-\alpha_{N}}+\alpha_{N} e^{-\alpha_{N}}+O\left(\frac{1}{N^{1-\delta}}\right), \\
& \mathbf{D} \mu_{0}\left(D_{1}\right)=N e^{-\alpha_{N}}\left(1-e^{-\alpha_{N}}\right)+O(1) .
\end{aligned}
$$

Для несплошных 3-цепочек $\left(v=3, r_{2}=r, r_{3}=s, 2 r \neq s+1\right)$ с множеством

$$
\mathscr{B}_{N}=D_{2}
$$

можно повторить вывод асимптотических формул, приведенный в [2] для сплошных цепочек. При этом существенно используются вероятности $\mathbf{P}(\bar{x}(t)=I, \bar{x}(t+u)=J)$.

Для несплошных цепочек вида (32) цепочки $\bar{x}(t)=\left(x_{t}, x_{t+r-1}, x_{t+s-1}\right)$ и $\bar{x}(t+u)=\left(x_{t+u}, x_{t+u+r-1}, x_{t+u+s-1}\right)$ сцеплены, если $u \in d=\{r-1, s-r, s-1\}$ и при этом они сцеплены ровно по одной координате. При выводе формул в [2] существенно также использовались множества $B_{1}(u), B_{2}(u)$ значений пар $(I, I)$ и $(I, J)$, при которых вероятности событий $\{\bar{x}(t)=I, \bar{x}(t+u)=J\}$ положительны. Определим аналогичные множества в рассматриваемом случае, полагая

$$
\begin{array}{ll}
B_{1}(r-1)=\left\{I: i_{2}=i_{1}, I \in D_{2}\right\}, & B_{1}(s-r)=\left\{I: i_{3}=i_{2}, I \in D_{2}\right\}, \\
B_{1}(s-1)=\left\{I: i_{3}=i_{1}\right\}, & B_{2}(r-1)=\left\{(I, J): I \neq J, i_{2}=j_{1}\right\}, \\
B_{2}(s-r)=\left\{(I, J): I \neq J, i_{3}=j_{2}\right\}, & B_{2}(s-1)=\left\{(I, J): I \neq J, i_{3}=j_{1}\right\} .
\end{array}
$$

При любом $u \in d$

$$
\left|B_{1}(u)\right|=N^{2}, \quad\left|B_{2}(u)\right| \leqslant N^{5}
$$

Отметим также, что в рассматриваемом случае

$$
\left|T_{1}(l)\right|=\frac{n^{l}}{l !}-\frac{7}{2} \frac{n^{l-1}}{(l-2) !}+O\left(l^{3} \frac{n^{l-2}}{(l-2) !}\right) .
$$

Используя эти замечания, можно повторить вывод асимптотических формул, приведенный в [2], для нашего случая.

Для несплошных 3-цепочек вида (32) при $N \rightarrow \infty$ в схеме серий (3)

$$
\begin{aligned}
& \mathbf{E} \mu_{0}\left(D_{2}\right)=N^{3} A_{1}^{*}(1)-\frac{7}{2} \alpha_{N} A_{1}^{*}\left(a^{2}(I)\right) \\
& +\alpha_{N}\left(\frac{1}{N^{2}} \sum_{h \in d} \sum_{I \in D_{2}} a_{h}(I, I) e^{-\alpha_{N} a(I)}\right) \\
& +O\left(\frac{1}{N^{1-\delta}}\right), \quad 0<\delta<1, \\
& \mathbf{D} \mu_{0}\left(D_{2}\right)=N^{3} \sigma_{N}^{2}\left(D_{2}\right)+O\left(N^{1+\delta}\right), \quad 0<\delta<1,
\end{aligned}
$$


где

$$
\begin{gathered}
\sigma_{N}^{2}\left(D_{2}\right)=A_{1}^{*}(1)-A_{2}^{*}(1)-7 \alpha_{N}\left(A_{1}^{*}(a(I))\right)^{2}+\frac{2 \alpha_{N}}{N^{5}} \sum_{h \in d} \sum_{I, J \in D_{2}} a_{h}(I, J) e^{-\alpha_{N}(a(I)+a(J))}, \\
a(I)=a_{i_{1}} a_{i_{2}} a_{i_{3}}, \\
A_{h}^{*}(b(I))=\frac{1}{N^{3}} \sum_{I \in D_{2}} b(I) e^{-h \alpha_{N} a(I)}, \quad h=1,2, \\
a_{r-1}(I, J)=a(I) a_{j_{2}} a_{j_{3}} \delta_{i_{2} j_{1}}, a_{s-r}(I, J)=a(I) a_{j_{1}} a_{j_{3}} \delta_{i_{3} j_{2}}, a_{s-1}(I, J)=a(I) a_{j_{2}} a_{j_{3}} \delta_{i_{3} j_{1}} .
\end{gathered}
$$

Формулы (35) и (36) при $p_{k}=1 / N, k=1, \ldots, N$, имеют вид

$$
\begin{aligned}
& \mathbf{E} \mu_{0}\left(D_{2}\right)=N^{3} e^{-\alpha_{N}}-\frac{\alpha_{N}}{2} e^{-\alpha_{N}}+O\left(\frac{1}{N^{1-\delta}}\right), \\
& \mathbf{D} \mu_{0}\left(D_{2}\right)=N^{3} e^{-\alpha_{N}}\left(1-\left(1+\alpha_{N}\right) e^{-\alpha_{N}}\right)+O\left(N^{1+\delta}\right), \quad 0<\delta<1 .
\end{aligned}
$$

Отметим, что в [2] приведены условия, при которых

$$
\frac{1}{\left|\mathscr{B}_{N}\right|} \mathbf{D} \mu_{0}\left(\mathscr{B}_{N}\right) \geqslant \sigma_{0}^{2}>0 \text {. }
$$

При этих условиях $\sigma_{N}^{2}\left(D_{1}\right)$ и $\sigma_{N}^{2}\left(D_{2}\right)$ также отделены от 0. Будем предполагать, что

$$
0<C_{1} \leqslant N p_{k} \leqslant C_{2}<\infty, \quad k=1, \ldots, N .
$$

Из этого ограничительного условия следует условие (39) работы [2].

Получим теперь асимптотические формулы для $\mathbf{D} \mu_{0}^{*}\left(D_{k}\right), k=1,2$. Положим

$$
\underline{\Delta}_{u}=\sum_{l=0}^{\varphi-1} \Delta_{u}(l), \quad u=2,3,
$$

где $\Delta_{u}(l)$ определено равенством (19).

Лемма 4. Если выполнены условия (39), то в схеме серий (3) при $N \rightarrow \infty$ для $\mathscr{B}_{N}=D_{2}$ или $\mathscr{B}_{N}=D_{3}$

$$
\lim _{N \rightarrow \infty} \frac{\mathbf{E} \Delta_{k}^{2}}{\mathbf{D} \mu_{0}\left(\mathscr{B}_{N}\right)}=0, \quad k=2,3 ; \quad \lim _{N \rightarrow \infty} \frac{\mathbf{E} \bar{\Delta}_{k}^{2}}{\mathbf{D} \mu_{0}\left(\mathscr{\Re}_{N}\right)}=0, \quad k=1,2,3,
$$

где $\underline{\Delta}_{k}, \bar{\Delta}_{k}$ определены равенствами (18), (40).

Доказательство. Доказательство, так же, как и доказательство предыдущей леммы, сводится к оценке числа слагаемых с различного вида пересечениями $v$-цепочек. Докажем утверждение леммы для $\underline{\Delta}_{2}^{2}$ в случае $\mathscr{B}_{N}=D_{1}$. Представим $\underline{\Delta}_{2}$ в виде

$$
\underline{\Delta}_{2}=\sum_{i=1}^{N} \sum_{l=2}^{\varphi-1} \sum_{\bar{t}(l) \in T_{2}(l)} \chi_{i}\left(t_{1}\right) \ldots \chi_{i}\left(t_{l}\right), \quad \chi_{i}(t)=\chi\left(x_{t}=\ldots=x_{t+s-1}=i\right) .
$$

Отсюда следует, что

$$
\mathbf{E}\left(\underline{\Delta}_{2}\right)^{2}=E_{1}+E_{2}
$$


где

$$
\begin{gathered}
E_{1}=\sum_{i \neq j} E(I, J), \quad E_{2}=\sum_{i=1}^{N} E(I, I), \quad I=(i, \ldots, i), \quad J=(j, \ldots, j), \\
E(I, J)=\sum_{l=4}^{2 \varphi-2} F_{l}(I, J), \quad F_{l}(I, J)=\sum_{u=0}^{l} \sum_{l} \sum_{l-u} \mathbf{E} \chi_{i}\left(t_{1}^{\prime}\right) \ldots \chi_{i}\left(t_{u}^{\prime}\right) \chi_{j}\left(t_{1}^{\prime \prime}\right) \ldots \chi_{j}\left(t_{l-u}^{\prime \prime}\right),
\end{gathered}
$$

в суммах $\sum_{l}$ и $\sum_{l-u}$ суммирование проводится по $\bar{t}^{\prime}(u)=\left(t_{1}^{\prime}, \ldots, t_{u}^{\prime}\right) \in T_{2}(u)$ и $\bar{t}^{\prime \prime}(l-u)=\left(t_{1}^{\prime \prime}, \ldots, t_{l-u}^{\prime \prime}\right) \in T_{2}(l-u)$. Цепочки с началами из $\bar{t}^{\prime}(u)$ могут пересекаться с цепочками, начала которых принадлежат $\bar{t}^{\prime \prime}(l-u)$. Если пересечение есть, то при $I \neq J$ соответствующие слагаемые равны 0 и, таким образом, остаются только слагаемые с непересекающимися цепочками с началами в $\bar{t}^{\prime}(u)$ и $\bar{t}^{\prime \prime}(l-u)$. Следовательно, для этих слагаемых

$$
\begin{aligned}
\mathbf{E} \chi_{i}\left(t_{1}^{\prime}\right) \ldots \chi_{i}\left(t_{u}^{\prime}\right) \chi_{j}\left(t_{1}^{\prime \prime}\right) \ldots \chi_{j}\left(t_{l-u}^{\prime \prime}\right) & =\mathbf{E} \chi_{i}\left(t_{1}^{\prime}\right) \ldots \mathbf{E} \chi_{i}\left(t_{u}^{\prime}\right) \mathbf{E} \chi_{j}\left(t_{1}^{\prime \prime}\right) \ldots \mathbf{E} \chi_{j}\left(t_{l-u}^{\prime \prime}\right) \\
& \leqslant \frac{A^{s(u-2)}}{N^{s(u-2)}} \frac{A^{s+1}}{N^{s+1}} \frac{A^{s(l-u-2)}}{N^{s(l-u-2)}} \frac{A^{s+1}}{N^{s+1}}=\left(\frac{A}{N}\right)^{s(l-2)}\left(\frac{A}{N}\right)^{2},
\end{aligned}
$$

так как сцепленные цепочки полностью не совпадают и наименьшая длина сцепленных цепочек из $T_{2}(u), T_{2}(l-u)$ не меньше $s+1$. Отсюда следует, что

$$
E_{1} \leqslant N(N-1) \sum_{l=4}^{\infty} \frac{n^{l-2}}{(l-2) !} s^{2} \frac{A^{s(l-2)}}{N^{s(l-2)}} \frac{A^{2}}{N^{2}} \leqslant A^{2} \sum_{l=4}^{\infty} \frac{\left(\alpha_{n} A\right)^{l-2}}{(l-2) !}<\infty .
$$

Слагаемые в суммах $E_{2}$, образованные сцепленными цепочками, положительны. Множество $s$-цепочек каждого слагаемого, входящего в сумму $F_{l}(I, J)$, разобьем на изолированные группы сцепленных цепочек. Пусть $m_{k}$ - число изолированных групп, состоящих из $k$ цепочек, $k=1, \ldots, l$. Тогда

$$
m_{1}+2 m_{2}+\ldots+l m_{l}=l, \quad m_{2}+\ldots+m_{l} \geqslant 1 .
$$

Число способов выбора $l$ начал $s$-цепочек, входящих в $F_{l}(I, J)$ с заданными $m_{1}, \ldots, m_{l}$, не превосходит

$$
\frac{n^{m_{1}}}{m_{1} !} \frac{n^{m_{2}} s}{m_{2} !} \ldots \frac{n^{m_{l}} s^{l-1}}{m_{l} !}
$$

Каждое слагаемое при заданных $m_{1}, \ldots, m_{l}$ не превосходит

$$
C\left(\frac{1}{N^{s}}\right)^{m_{1}+\ldots+m_{l}} \frac{1}{N}
$$

где $C-$ положительная постоянная. Из этих оценок следует, что

$$
E_{2} \leqslant N \sum_{l=4}^{2 \varphi-2} s^{l} \sum^{*} \frac{\left(\alpha_{N} A\right)^{m_{1}}}{m_{1} !} \ldots \frac{\left(\alpha_{N} A\right)^{m_{l}}}{m_{l} !} \frac{1}{N}
$$

где суммирование в $\sum^{*}$ проводится по всем $m_{1}, \ldots, m_{l}$, удовлетворяющим ограничениям (43). Заменив суммирование с ограничениями на суммирование по всем натуральным 
$m_{1}, \ldots, m_{l}$, получим, что

$$
E_{2} \leqslant \sum_{0=0}^{2 \varphi-2} s^{l}\left(\sum_{m=0}^{\infty} \frac{\left(\alpha_{N} A\right)^{m}}{m !}\right)^{l} \leqslant C_{1}\left(s e^{\alpha_{N} A}\right)^{2 \varphi} \leqslant C_{2} N^{\varepsilon_{N}},
$$

где $C_{1}, C_{2}-$ некоторые положительные постоянные, $\varepsilon_{N} \rightarrow 0$ при $N \rightarrow \infty$. Из оценок (42) и (44) следует, что при $N \rightarrow \infty$

$$
\mathbf{E} \underline{\Delta}_{2}^{2}=O\left(N^{\varepsilon_{N}}\right), \quad \mathbf{E} \underline{\Delta}_{2}^{2} / \mathbf{D} \mu_{0}(N) \rightarrow 0 .
$$

Остальные утверждения леммы доказываются аналогично.

Лемма 5. Если выполнены условия (39), то в схеме серий (3) с множеством $\mathscr{B}_{N}=D_{1}$ или $\mathscr{B}_{N}=D_{2}$

$$
\lim _{N \rightarrow \infty} \frac{\mathbf{D} \Delta_{\varphi}}{\mathbf{D} \mu_{0}\left(\mathscr{B}_{N}\right)}=0
$$

Доказательство. Из неравенства (18) и неравенств

$$
\mathbf{D} \Delta_{\varphi} \leqslant \mathbf{E} \Delta_{\varphi}^{2}+\left(\mathbf{E} \Delta_{\varphi}\right)^{2} \leqslant 2 \mathbf{E} \Delta_{\varphi}^{2}, \mathbf{E}\left(\bar{\Delta}_{u_{1}}, \bar{\Delta}_{u_{2}}\right) \leqslant \sqrt{\mathbf{E} \bar{\Delta}_{u_{1}}^{2} \mathbf{E} \bar{\Delta}_{u_{2}}^{2}}
$$

следует, что

$$
\mathbf{D} \Delta_{\varphi} \leqslant 2 \sum_{u_{1}, u_{2}=1}^{3} \sqrt{\mathbf{E} \bar{\Delta}_{u_{1}}^{2} \mathbf{E} \bar{\Delta}_{u_{2}}^{2}}=2\left(\sum_{u=1}^{3} \sqrt{\mathbf{E} \bar{\Delta}_{u}^{2}}\right)^{2} .
$$

Отсюда и из леммы 4 получаем, что

$$
\frac{\mathbf{D} \Delta_{\varphi}}{\mathbf{D} \mu_{0}\left(\mathscr{B}_{N}\right)} \leqslant 2\left(\sum_{u=1}^{3} \sqrt{\frac{\mathbf{E} \bar{\Delta}_{u}^{2}}{\mathbf{D} \mu_{0}\left(\mathscr{B}_{N}\right)}}\right) \rightarrow 0
$$

при $N \rightarrow \infty$.

Теорема 3. Если выполнены условия (39), то в схеме серий (3) при $N \rightarrow \infty$ с $\mathscr{B}_{N}=D_{1}$ или $\mathscr{B}_{N}=D_{2}$

$$
\lim _{N \rightarrow \infty} \frac{\mathbf{D} \mu_{0}^{*}\left(\mathscr{B}_{N}\right)}{\mathbf{D} \mu_{0}\left(\mathscr{B}_{N}\right)}=1 .
$$

Доказательство. Поделим на $\mathbf{D} \mu_{0}(N)$ обе части равенства

$$
\mathbf{D} \mu_{0}\left(\mathscr{B}_{N}\right)=\sum_{u_{1}, u_{2}=1}^{4} \operatorname{cov}\left(\mu_{u_{1}}(N), \mu_{u_{2}}(N)\right),
$$

где $\mu_{1}(N)=\mu_{0}^{*}\left(\Re_{N}\right), \mu_{4}(N)=\Delta_{\varphi}$. Из получившегося равенства, используя неравенства

$$
\begin{aligned}
\operatorname{cov}\left(\mu_{u_{1}}(N), \mu_{u_{2}}(N)\right) & \leqslant \sqrt{\mathbf{D} \mu_{u_{1}}(N) \mathbf{D} \mu_{u_{2}}(N),} \\
\mathbf{D} \mu_{u}(N) & \leqslant 2 \mathbf{E} \underline{\Delta}_{u}^{2}
\end{aligned}
$$

и леммы 4 и 5 , получим, что

$$
1=\frac{\mathbf{D} \mu_{1}(N)}{\mathbf{D} \mu_{0}\left(\mathscr{B} c_{N}\right)}+\gamma_{1}(N) \sqrt{\frac{\mathbf{D} \mu_{1}(N)}{\mathbf{D} \mu_{0}\left(\mathscr{B}_{N}\right)}}+\gamma_{2}(N),
$$

где $\gamma_{1}(N) \rightarrow 0, \gamma_{2}(N) \rightarrow 0$ при $N \rightarrow \infty$. Отсюда следует утверждение теоремы. 
Приведем две теоремы об асимптотической нормальности $\mu_{0}\left(\mathscr{B}_{N}\right)$.

Теорема 4. Пусть случайная величина $\mu_{0}\left(\mathscr{B}_{N}\right)$ определена по сплошным v-цепочкам (2) $c v=s u$

$$
\mathscr{B}_{N}=D_{1}=\left\{\left(i_{1}, \ldots, i_{s}\right): i_{1}=\ldots=i_{s}=i, i=1, \ldots, N\right\},
$$

и пусть выполнены условия (39). Тогда в схеме серий (3) распределение случайных величин

$$
\frac{\mu_{0}\left(\mathscr{B}_{N}\right)-N A_{1}(1)}{\sigma_{N}\left(D_{1}\right) \sqrt{N}}
$$

при $N \rightarrow \infty$ сходится к стандартному нормальному распределению, $A_{l}(1), \sigma_{N}^{2}\left(D_{1}\right)$ определень равенствами (30).

Теорема 5. Пусть случайная величина $\mu_{0}\left(\mathscr{B}_{N}\right)$ определена по несплошным v-цепочкам (2) $c v=3, r_{2}=r, r_{3}=s, r \neq 2 s-1 u$

$$
\mathscr{B}_{N}=D_{2}=\left\{\left(i_{1}, i_{2}, i_{3}\right): i_{k}=1, \ldots, N, k=1,2,3\right\}
$$

и выполнены условия (39). Тогда в схеме серий (3) распределение случайных величин

$$
\frac{\mu_{0}\left(\mathscr{B}_{N}\right)-N^{3} A_{1}^{*}(1)}{\sigma_{N}\left(D_{2}\right) \sqrt{N}}
$$

$n р и \quad N \rightarrow \infty$ сходится к стандартному нормальному распределению, $A_{1}^{*}(1), \sigma_{N}^{*}\left(D_{2}\right)$ определены равенством (37).

Утверждения этих теорем следуют из теоремы 2, условия которой в рассматриваемых случаях проверяются при помощи теоремы 3 , асимптотических формул для $\mathbf{E} \mu_{0}\left(\mathscr{B}_{N}\right)$, $\mathbf{D} \mu_{0}\left(\mathscr{B}_{N}\right)$, лемм 1-3 и равенства

$$
\mathbf{E} \mu_{0}\left(\mathscr{B}_{N}\right)-\mathbf{E} \mu_{1}(N)=\mathbf{E} \mu_{2}(N)+\mathbf{E} \mu_{3}(N)+\mathbf{E} \Delta_{\varphi} .
$$

\section{Список литературы}

1. Тихомирова М. И., Об асимптотической нормальности числа непоявившихся $s$-цепочек. Дискретная математика (1992) 4, №2, 122-129.

2. Тихомирова М. И., Чистяков В. П., Об асимптотике моментов числа непоявившихся $s$-цепочек. Дискретная математика (1997) 9, №1, 11-21.

3. Тихомирова М. И., Предельные теоремы для числа непоявивиихся s-цепочек, Диссертация на соискание ученой степени кандидата физико-математических наук. МИЭМ, Москва, 1998.

4. Михайлов В. Г., Асимптотическая нормальность разделимых статистик от частот $m$-цепочек. Дискретная математика (1989) 1, №4, 92-103.

5. Тихомирова М. И., Предельные распределения числа непоявившихся цепочек одинаковых исходов. Дискретная математика (2008) 20, №3, 40-46.

6. Феллер В., Введение в теорию вероятностей и ее приложения, 1. Мир, Москва, 1984.

7. Бернштейн С. Н., Собрание сочинений, 4. Наука, Москва, 1964.

8. Janson S., Normal convergence by higher semiinvariants with application to sums of dependent random variables and random graphs. Ann. Probab. (1988) 16, №1, 305-312.

9. Михайлов В. Г., Об одной теореме Янсона. Теория вероятностей и ее применения (1991) 36, $168-170$. 